\title{
Genotype-phenotype correlation in a family with late onset CMT and an MPZ lys236del mutation
}

\author{
J E Sowden, E L Logigian, K Malik, D N Herrmann
}

J Neurol Neurosurg Psychiatry 2005;76:442-444. doi: 10.1136/jnnp.2004.043968

An in frame, lys236 deletion in the intracytoplasmic domain of myelin protein zero (MPZ) has recently been designated as a mutation possibly associated with Charcot-Marie-Tooth disease (CMT) but requiring further documentation. In this report we present a detailed clinical, electrophysiological, and genotype correlation in three generations of a family with the MPZ lys236del mutation and provide further evidence that this mutation is associated with CMT. The MPZ lys236del mutation is associated with an autosomal dominant, adult onset CMT phenotype, with variable penetrance ranging from an asymptomatic state to foot deformities, pedal numbness, and muscle cramps. Nerve conduction studies disclose intermediate range, somewhat non-uniform slowing of motor nerve conduction, which is accentuated in forelimb rather than distal nerve segments. Based on the contrasting finding of entirely normal conduction velocities $(\mathrm{CV})$ in a genetically affected 15 year old in this family, it remains to be established whether CV slowing with this mutation is progressive in life, a pattern that would contrast with CMT1a (PMP22 gene duplication).

M yelin protein zero (MPZ) gene mutations result in diverse, autosomal dominant Charcot-Marie-Tooth (CMT) neuropathy phenotypes. ${ }^{12}$ A severe early onset phenotype with very slow conduction velocities (CV) has been associated with mutations that disrupt the tertiary structure of MPZ and interfere with myelin compaction. ${ }^{2}$ An adult onset phenotype with mildly slowed or normal CV and more prominent axonal degeneration has been associated with mutations that are suggested to impair axon-schwann cell interactions. ${ }^{3}$ While $109 \mathrm{MPZ}$ gene mutations have been reported in association with CMT (http://www.molgen. ua.ac.be/CMTMutations/default.cfm), only three have been in frame, single codon deletions, most being due to point substitutions of a single nucleotide. Recently Street et al described a single codon deletion of the intracellular domain of MPZ (MPZ lys236del) in two members of a family with mild late onset CMT. ${ }^{3}$ The authors designated this codon deletion as a probable disease causing mutation, requiring further documentation, given the in frame nature of the deletion. ${ }^{3}$

Here we provide additional evidence, in another family, that the MPZ lys236del mutation is associated with a mild late onset CMT phenotype with variable penetrance, and we describe detailed clinical, electrophysiological, and genotype correlations in three generations of this family.

PROBAND (II.2)

A 47 year old man presented with a 2 year history of progressive numbness of both feet. He noted lifelong nocturnal cramps in the feet and lower legs that had increased in frequency over a 1 year period, and high arched feet and hammertoes since childhood. Neurological examination showed pes cavus, hammertoes, mild atrophy of intrinsic foot muscles, normal strength, and diffusely unobtainable deep tendon reflexes. Vibration sensation was absent at the toes and pinprick sensation was reduced to the ankles, while proprioception was normal. He had mild difficulty with tandem gait.

Nerve conduction studies (NCS) (table 1) showed reduced amplitude peroneal $(0.6 \mathrm{mV})$ and tibial $(1.1 \mathrm{mV})$ compound muscle action potentials (CMAP) with mildly prolonged distal latencies (DL) (7.8 and 7.1 ms) but more prominent CV slowing ( 24 and $25 \mathrm{~m} / \mathrm{s}$ ). Ulnar and median motor studies showed normal CMAP amplitudes ( 7.5 and $9.5 \mathrm{mV}$ ) with mildly prolonged DL (3.9 and $5.2 \mathrm{~ms}$ ) but slower CV (33 and $29 \mathrm{~m} / \mathrm{s}$ ). The sural sensory nerve action potential (SNAP) was absent, while upper extremity SNAPs were of low amplitude with moderately reduced CV. Needle examination of the tibialis anterior muscle showed reinnervation but no active denervation. Laboratory testing showed normal blood glucose, creatinine, ESR, serum protein electrophoresis and immunofixation, and vitamin B12 level.

\section{FAMILY STUDIES}

\section{Proband's father}

The proband's father (I.1), a 71 year old man, described frequent "ankle sprains", leg cramps, high arched feet, and hammertoes, and repeated unexplained dislocations of his knees since childhood. He had no sensory complaints. Examination showed pes cavus and hammertoes. Muscle strength was normal except for mild toe flexion weakness; ankle reflexes were absent but knee reflexes were normal. Vibration sensation, proprioception, and gait were normal. NCS showed a low amplitude peroneal CMAP with a mildly prolonged DL and reduced CV $(30 \mathrm{~m} / \mathrm{s})$. The median CMAP amplitude was normal with a DL of $5.7 \mathrm{~ms}$ and CV of $39 \mathrm{~m} / \mathrm{s}$. The sural SNAP was unobtainable.

\section{Proband's brother (II.3)}

A 43 year old man had a history of a remote lumbar disc herniation with residual sensory loss over the left lateral foot and calf but no generalised peripheral neuropathic complaints. Examination showed an asymptomatic decrease in hearing (left worse than right) with a sensorineural pattern clinically. The heel cords were mildly shortened, but there were no foot deformities. Strength was normal. Ankle reflexes were absent on the left and trace on the right. Vibration sensation was equivocally reduced at the toes, but proprioception, pinprick sensation, and gait were normal. NCS showed a polyneuropathy with disproportionate slowing of CV. The peroneal CMAP amplitude was normal, DL was

Abbreviations: CMAP, compound muscle action potentials; CMT, Charcot-Marie-Tooth disease; CV, conduction velocity; DL, distal latency; MPZ, myelin protein zero; NCS, nerve conduction studies; SNAP, sensory nerve action potential 
Table 1 Electrophysiologic findings in the proband and family members

\begin{tabular}{|c|c|c|c|c|c|c|c|}
\hline \multirow[b]{2}{*}{ Patient } & \multirow[b]{2}{*}{ Age (years) } & \multirow{2}{*}{$\begin{array}{l}\text { Lys } 236 \text { del } \\
\text { (yes/no) }\end{array}$} & \multicolumn{5}{|c|}{ Nerve conduction studies } \\
\hline & & & Nerve (ms) & Amp $m \mathrm{~m}(\mathrm{M})(\mu \mathrm{V})(\mathrm{S})$ & $\mathrm{DL}$ (ms) & $\mathrm{CV}(\mathrm{m} / \mathrm{s})$ & F wave lat (ms) \\
\hline \multirow{6}{*}{$\begin{array}{l}\text { Il.2 } \\
\text { (proband) }\end{array}$} & 47 & Yes & Peroneal & $0.56(\geqslant 2.0)$ & $7.8(\leqslant 6.5)$ & $24(\geqslant 41)$ & $N D(\leqslant 56)$ \\
\hline & & & Tibial & $1.1(\geqslant 4.0)$ & $7.1(\leqslant 5.8)$ & $25(\geqslant 41)$ & $106.7(\leqslant 56)$ \\
\hline & & & Median (M) & $9.5(\geqslant 4.0)$ & $5.2(\leqslant 4.4)$ & $29(\geqslant 49)$ & $N D(\leqslant 31)$ \\
\hline & & & Ulnar & $7.5(\geqslant 6.0)$ & $3.9(\leqslant 3.5)$ & $33(\geqslant 49)$ & $46.5(\leqslant 32)$ \\
\hline & & & Sural & $N R(\geqslant 7)$ & & $N R(\geqslant 41)$ & \\
\hline & & & Median (s) & $7.4(\geqslant 20)$ & & $41(\geqslant 50)$ & \\
\hline \multirow[t]{4}{*}{1.1} & 71 & Yes & Peroneal & $0.5(\geqslant 2.0)$ & $7.6(\leqslant 6.5)$ & $30(\geqslant 41)$ & $74.1(\leqslant 56)$ \\
\hline & & & Median (M) & $8.2(\geqslant 4.0)$ & $5.7(\leqslant 4.4)$ & $39(\geqslant 49)$ & $35.6(\leqslant 31)$ \\
\hline & & & Sural & $N R(\geqslant 2.5)$ & & $N R(\geqslant 41)$ & \\
\hline & & & Median (S) & $9.8(\geqslant 15)$ & & $39(\geqslant 50)$ & \\
\hline \multirow[t]{4}{*}{1.2} & 68 & No & Peroneal & $3.9(\geqslant 2.0)$ & $4.0(\leqslant 6.5)$ & $46(\geqslant 41)$ & $44.2(\leqslant 56)$ \\
\hline & & & Median (M) & $11.1(\geqslant 4.0)$ & $3.1(\leqslant 4.4)$ & $54(\geqslant 49)$ & $26.7 i \leqslant 31)$ \\
\hline & & & Sural & $6.5(\geqslant 4.5)$ & & $40(\geqslant 41)$ & \\
\hline & & & Median (S) & $20.7(\geqslant 15)$ & & $59(\geqslant 50)$ & \\
\hline \multirow[t]{4}{*}{ 11. 3} & 43 & Yes & Peroneal & $2.8(\geqslant 2.0)$ & $5.8(\leqslant 6.5)$ & $28(\geqslant 41)$ & $67.6(\leqslant 56)$ \\
\hline & & & Median (M) & $9.2(\geqslant 4.0)$ & $4.3(\leqslant 4.4)$ & $47(\geqslant 49)$ & $31.4(\leqslant 31)$ \\
\hline & & & Sural & $8.9(\geqslant 6)$ & & $34(\geqslant 41)$ & \\
\hline & & & Median (S) & $28.3(\geqslant 20)$ & & $46(\geqslant 50)$ & \\
\hline \multirow[t]{4}{*}{ II. 4} & 32 & No & Peroneal & $11.2(\geqslant 2.0)$ & $5.1(\leqslant 6.5)$ & $45(\geqslant 41)$ & $50.1(\leqslant 56)$ \\
\hline & & & Median (M) & $7.9(\geqslant 4.0)$ & $3.4(\leqslant 4.4)$ & $60(\geqslant 49)$ & $29.8(\leqslant 31)$ \\
\hline & & & Sural & $7 . .3(\geqslant 6)$ & & $46(\geqslant 41)$ & \\
\hline & & & Median (S) & $28.8(\geqslant 20)$ & & $59(\geqslant 50)$ & \\
\hline \multirow[t]{4}{*}{ III. 1} & 15 & Yes & Peroneal & $7.8(\geqslant 2.0)$ & $6.4(\leqslant 6.5)$ & $43(\geqslant 41)$ & $50.9(\leqslant 56)$ \\
\hline & & & Median (M) & $8.3(\geqslant 4.0)$ & $3.5(\leqslant 4.4)$ & $56(\geqslant 49)$ & $26.7(\leqslant 31)$ \\
\hline & & & Sural & $21.5(\geqslant 6)$ & & $46(\geqslant 41)$ & \\
\hline & & & Median (S) & $76.7(\geqslant 20)$ & & $57(\geqslant 50)$ & \\
\hline \multirow[t]{4}{*}{ III.2 } & 13 & No & Peroneal & $7.50(\geqslant 2.0)$ & $5.7(\leqslant 6.5)$ & $43(\geqslant 41)$ & $50.5(\leqslant 56)$ \\
\hline & & & Median (M) & $12.3(\geqslant 4.0)$ & $3.3(\leqslant 4.4)$ & $60(\geqslant 49)$ & $26.0(\leqslant 31)$ \\
\hline & & & Sural & $20.8(\geqslant 6)$ & & $45(\geqslant 41)$ & \\
\hline & & & Median (S) & $46.5(\geqslant 20)$ & & $57(\geqslant 50)$ & \\
\hline
\end{tabular}

Normal values are shown in parentheses. Amp, amplitude; CV, conduction velocity; DL, distal latency; lat, latency; M, motor; ND, not done; NR, no response; S, sensory.

normal $(5.8 \mathrm{~ms})$, but peroneal CV was $28 \mathrm{~m} / \mathrm{s}$. The median CMAP amplitude and DL were normal but the CV was slightly slowed. The sural SNAP had normal amplitude but slowed CV $(34 \mathrm{~m} / \mathrm{s})$.

\section{Proband's daughter (III.1)}

The proband's daughter was a 15 year old girl with no neuropathic complaints. Her parents reported that she had always been "pigeon toed". Examination revealed normal strength and deep tendon reflexes and no foot deformities. Vibration sense at the toes and index finger appeared mildly reduced on bedside testing considering her age. Proprioception, pinprick testing, and gait were normal. Quantitative sensory testing (CASE IV system, WR Medical Electronics, Stillwater, MN) confirmed that vibration thresholds were elevated at the great toe (99th percentile) and index finger (99th percentile), while cooling thresholds were normal at the foot (77th percentile) and index finger (57th percentile). NCS were normal (table 1).

The proband's mother (I.2), youngest brother (II.4), and youngest daughter (III.2) had no symptoms or signs of peripheral neuropathy, no foot deformities, and normal NCS (table 1)

\section{GENETIC STUDIES}

Genetic testing performed as part of clinical testing (Athena Diagnostics, Worcester, MA) on the proband showed no sequence alteration in PMP 22, early growth response 2, neurofilament-L, or Connexin 32 genes. MPZ gene sequencing showed an in frame, $3 \mathrm{bp}$ AAG deletion at codon position 236, resulting in a lys236del mutation in the MPZ protein.

Further genetic testing performed on a research basis on the proband and family members (under a local IRB approved protocol and after informed consent), using PCR primers (oligonucleotides) amplifying a $456 \mathrm{bp}$ region spanning exons V and VI and containing the 3 bp AAG codon deletion, confirmed the heterozygous presence of the MPZ lys236del mutation in the proband and in the clinically and electrophysiologically affected father (I.1) and brother (II.3) of the proband (fig 1). The proband's 15 year old daughter (III.1), who had no symptoms, foot deformities, or electrophysiologic abnormalities (but in whom vibration sense was abnormal), was found to also carry the deletion. Family members I.2, II.4, and III.2, who were clinically and

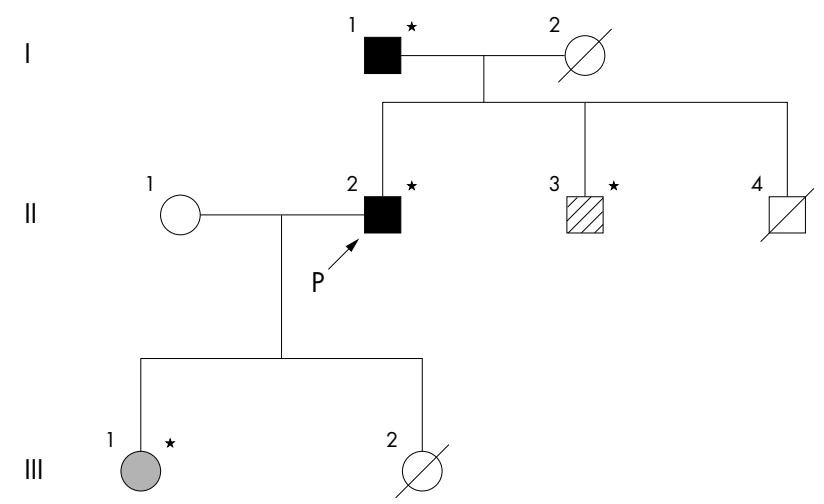

Figure 1 Family pedigree. The proband is member II.2. All family members except II. 1 were examined clinically and electrophysiologically and had genetic testing carried out. Squares are males, circles are females; * indicates individuals with a heterozygous MPZ lys 236 del; family members with signs and symptoms and electrophysiologic evidence of CMT are shown in black; hatched shading denotes asymptomatic CMT with electrophysiologic abnormalities; grey shading indicates an asymptomatic affected state with vibration sense abnormalities on quantitative testing but no electrophysiologic evidence of CMT; diagonal lines show clinically, electrophysiologically, and genetically unaffected family members. 
electrophysiologically normal, and 102 control DNA samples did not show the deletion.

\section{DISCUSSION}

This report provides further evidence of an association between a $3 \mathrm{bp}$ (AAG) deletion in the intracytoplasmic domain of MPZ (lys236del) and CMT disease. This deletion produces a mild CMT phenotype with variable presence of foot deformities, leg cramps, and pedal numbness. Although the presentation is a late onset one, certain symptoms, most consistently lower limb cramps, are present from childhood. Patients with this deletion demonstrate variable clinical and electrophysiologic penetrance, and an intermediate pattern of $\mathrm{CV}$ slowing that is non-uniform, being accentuated in forelimb as compared to distal motor nerve segments.

It is uncertain how the MPZ lys236del mutation leads to a CMT phenotype, however a lys236 is conserved between species suggesting functional importance. ${ }^{3}$ The intracytoplasmic domain of MPZ is felt to facilitate myelin compaction through involvement in an adhesion mediated signal transduction cascade. ${ }^{23}$ The lys236del mutation may alter the phosphorylation state of serine residues located near the carboxy terminus of MPZ which have been suggested to be important in regulating the role of MPZ in myelin compaction. $^{3}$ Dysregulation of myelin compaction may account for the observed slowing of CV in this family. The axon loss seen in the proband suggests that the lys236del mutation also affects the role of the intracytoplasmic domain of MPZ in maintaining schwann cell-axon relationships. ${ }^{2}$

The phenotype-genotype correlations raise issues of clinical importance for II.3, who at age 43 was asymptomatic and had no foot deformities, and might in a clinical setting have been mistaken for having an acquired peripheral neuropathy. II.3 and other affected adults in the family showed non-uniform conduction slowing with disproportionate slowing of motor CV versus milder DL slowing (median nerve excepted). Accentuation of CV slowing in intermediate nerve segments was also evident in the MPZ lys236del mutation index case of Street et al and has been noted in Japanese kindreds with other MPZ mutations and late onset CMT. ${ }^{3}$ This electrophysiologic pattern contrasts with that seen in another nonuniform hereditary neuropathy (hereditary neuropathy with liability to pressure palsies) where distally accentuated slowing is typical but resembles that seen in diabetic distal polyneuropathy. ${ }^{56}$ Careful analysis of electrophysiologic data from additional late onset MPZ mutations is required to see whether this pattern of slowing is common and distinctive among the hereditary neuropathies.

Family member III.1, who at age 15 was asymptomatic, had no deformities, and had a normal neurologic examination except for mildly reduced vibration sensation, is informative. III.l carries the lys236del mutation but, in contrast to the affected adults, has normal NCS. While the clinical phenotype of late onset MPZ mutations shows significant intra-family variability, the electrophysiological phenotype has, in contrast to our family, been reported to show less within family variation. ${ }^{4}$ A median motor CV of $39 \mathrm{~m} / \mathrm{s}$ has been used to distinguish "demyelinating" and "axonal" phenotypes of CMT, based primarily on studies in CMTla. ${ }^{4}$ While this CV value is useful to predict the CMTla genotype and some MPZ mutations, ${ }^{4}$ our study suggests that this cut off value is not helpful in predicting an MPZ lys236del mutation.

Longitudinal studies in CMTla with PMP 22 duplications have shown that CV slowing is established from early childhood and that CV remains stable in patients followed over a 20 year period, despite progressive axon loss. ${ }^{5}$ The stability of motor CV with time is less well studied in MPZ mutations. ${ }^{5}$ One small cross sectional study has suggested that CV do not vary significantly with age with MPZ mutations. ${ }^{4}$ The normal NCS in the genetically affected daughter (III.1) of the proband suggest the possibility of an age dependent decline in CV in subjects with the MPZ lys236del mutation or alternatively a gender effect, although such an effect has not previously been reported with MPZ mutations. The case of III.l emphasises that in families with late onset MPZ mutations, electrophysiologic testing early in life may be insufficient to determine whether a family member is or will be affected, or not.

Finally, age and gender alone cannot account for the phenotypic variability observed in this family, given the more severe phenotype of the proband as compared with II.3, who is about the same age. It is likely that there are additional genetic influences upon the phenotype in individuals with the MPZ lys236del mutation.

\section{ACKNOWLEDGEMENTS}

Laurie Aronberg and Valerie Pannoni are acknowledged for assistance with manuscript preparation, Chris Blood for study coordination, and Marlene Hogan and Michele Ferguson for technical assistance.

\section{Authors' affiliations}

J E Sowden, E L Logigian, K Malik, D N Herrmann, Department of Neurology, University of Rochester, Rochester, NY, USA

Competing interests: none declared

Correspondence to: David N Herrmann, MB, BCh, Department of Neurology, Box 673, University of Rochester Medical Center, 601 Elmwood Ave, Rochester, NY 14642, USA; david_herrmann@urmc. rochester.edu

Received 21 April 2004

In revised form 27 July 2004

Accepted 30 July 2004

\section{REFERENCES}

1 Roa BB, Warner LE, Garcia CA, et al. Myelin protein zero (MPZ) gene mutations in nonduplication type 1 Charcot-Marie-Tooth disease. Hum Mutat 1996;7(1):36-45

2 Shy ME, Jani A, Krajewski K, et al. Phenotypic clustering in MPZ mutations. Brain 2004; 127:371-84.

3 Street VA, Meekins G, Lipe HP, et al. Charcot-Marie-Tooth neuropathy: clinical phenotypes of four novel mutations in the MPZ and Cx 32 genes. Neuromuscul Disord 2002;12:643-50.

4 Hattori N, Yamamoto M, Yoshihara T, et al. Demyelinating and axonal features of Charcot-Marie-Tooth disease with mutations of myelin-related proteins (PMP22, MPZ and Cx32): a clinicopathological study of 205 Japanese patients. Brain 2003;126:134-51.

5 Lewis RA, Sumner AJ, Shy ME. Electrophysiological features of inherited demyelinating neuropathies: a reappraisal in the era of molecular diagnosis. Muscle Nerve 2000;23:1472-87.

6 Herrmann DN, Ferguson ML, Logigian EL. Conduction slowing in diabetic distal polyneuropathy. Muscle Nerve 2002;26:232-7. 